\title{
Tuning catalytic selectivity in cascade reactions by light irradiation
}

\author{
Xingguang Zhang, ${ }^{[\mathrm{a}]}$ Jianfeng $\mathrm{Yao}^{\left[{ }^{[\mathrm{a}]}\right.}$ and Xuebin $\mathrm{Ke}^{\star[\mathrm{b}]}$
}

Abstract: Photocatalytic synthesis using visible light offers a promising progress for clean chemicals synthesis because of its potential to harvest the abundant sunlight. Supported gold nanoparticles (Au-NPs) have been discovered to be efficient plamonic photocatalysts for selective reduction and oxidation reactions. This study highlight that the selectivity of cascade redox reactions (the selective reduction of nitrobenzene and the selective oxidation of benzyl alcohol) can be tuned via light irradiation over plasmonic $\mathrm{Au} / \mathrm{CeO}_{2}$. The net contribution of light irradiation correlates with the ability of incident light to excite electrons and light absorption of catalysts, which subsequently affect the reaction pathway and mechanism, thereby determining the final product selectivity.

Controlling selectivity in clean chemicals synthesis is crucial to obtaining target products with high purity, ${ }^{[1],[2]}$ and it is more challenging in cascade reactions. ${ }^{[3],[4]}$ Apart from optimizing reaction kinetics and mass transfer kinetics to achieve better catalytic efficiency, ${ }^{[5]}$ developing new catalysts by controlling active sites (e.g. composition, orientation, and distribution), ${ }^{[6],[7]}$ and by constructing nanostructures (e.g. pores, sizes, and shapes).[1], [8] Electrons (e.g. density, interaction, and transfer) also play an indispensable role in manipulating catalytic performances, particularly in photocatalysis which involves light-excited electrons. [9], [10] In parallel, strategies associated with energy input are developed to enhance product selectivity, such as the use of microwave, ultrasound, extra-electric or electromagnetic field, and light irradiation. [11], [12], [13]

Photocatalysis offers a promising opportunity to obtain a high product selectivity as its capacity to influence catalytic reactions at ambient temperatures. More significantly, the unique property of incident light interacting with reactants or photocatalysts is able to initiate or accelerate reactions with low energy barriers and to give a certain product.[14],[15],[16],[17] Recent studies demonstrate that plasmonic photocatalysts based on the localized surface plasmon resonance (LSPR) effect can actually achieve these goals. ${ }^{[18],[19],[20]}$ The LSPR effect features a collective and coherent oscillation of the free conduction electrons with the incident photons on the surface of metal nanoparticles (NPs).[21],[22],[23]These strong oscillating electrons can drive the target reaction locally (around active

\footnotetext{
[a] Dr. X.G. Zhang, Dr. J.F. Yao

College of Chemical Engineering

Nanjing Forestry University

No. 159, Longpan Road, Nanjing 210037, P.R.China

[b] Dr. X. B. Ke

School of Engineering and Computer Science

University of Hull

HU6 7 RX, United Kingdom.

Email: x.ke@hull.ac.uk
}

sites) and affect the product selectivity fundamentally. [24], [25] However, the functional mechanism of light irradiation to tune reactive procedure remains ambiguous.

Herein, a tuneable laser source and a simulate solar source are used to explore the processing mechanism involving incentive light, light absorption, electron excitation, electron transfer and reactants activation to obtain targeted products. In clean chemicals synthesis, the catalytic hydrogenation of nitro compounds is an ideal process to produce amines, ${ }^{[14]}$ and the catalytic oxidation of benzyl alcohol plays a significant role to produce aromatic aldehydes.[26] Conventional catalytic processes of these two consecutive reaction always necessitate complicated methodologies to minimize the production of side-products.[27],[28],[29],[30] This study highlights that light irradiation over supported plasmonic gold catalysts can effectively tune the catalytic selectivity of the aforementioned two cascade reactions, with the net contribution depending upon light wavelength and intensity at a certain temperature.

Table 1. Catalytic performances of $3 \% \mathrm{Au} / \mathrm{CeO}_{2}$ for selective reduction of nitrobenzene under the simulate sunlight source

\begin{tabular}{llllllll}
\hline & \\
\hline
\end{tabular}

a Product 1: azoxybenzene, ${ }^{\text {b} P r o d u c t ~ 2: ~ a z o b e n z e n e, ~}{ }^{\text {cPoduct } 3 \text { : }}$ aniline, ${ }^{\mathrm{d} C o n v e r s i o n . ~ R e a c t i o n ~ c o n d i t i o n s: ~ T i m e ~(6 h), ~}$ isopropanol $(30 \mathrm{ml})$, catalyst $(100 \mathrm{mg})$, nitrobenzene $(3 \mathrm{mmol})$, $\mathrm{KOH}(0.1 \mathrm{M}, 3 \mathrm{ml})$, light wavelength (Full), and light intensity (100 $\left.\mathrm{mW} \cdot \mathrm{cm}^{-2}\right)$. Control experiments: no products were detectable without catalysts or using the $\mathrm{CeO}_{2}$ support as catalysts under light on and off.

The catalytic performances of the hydrogenation of nitrobenzene are presented in Table 1. The effect of light irradiation on tuning the product selectivity is evident. In particular, at $40^{\circ} \mathrm{C}$ and with the light off, the selectivity of azoxybenzene (P1) can be up to $91 \%$, whereas with the light on, azoxybenzene (P1) disappears and the yield of azobenzene (P2) can be up to $>99 \%$. At a higher temperature $\left(80^{\circ} \mathrm{C}\right)$, the influence of light is negligible and both light reaction and thermal reaction can achieve a complete conversion. Generally, the light irradiation can increase the reaction conversion compared with the reaction with light off at lower reaction temperatures and have significant influence on 
product selectivity, thereby playing a threshold/switch role in tuning the catalytic reaction procedure.

To explain this phenomenon, the physiochemical properties of catalysts are characterized by transmission electron microscopy (TEM), X-ray photoelectron spectroscopy (XPS), and UV/Vis spectroscopy. In Figure 1a, the typical TEM images of the Au-NPs on the $\mathrm{CeO}_{2}$ support show that Au NPs are well dispersed and have narrow size distributions within 2-6 $\mathrm{nm}$. The XPS spectra (Figure 1b) provides the spectra of Au $4 \mathrm{f}$ and confirms that the binding energies of $A u 4 f_{7 / 2}$ and $A u 4 f_{5 / 2}$ electrons are 84.0 and $87.7 \mathrm{eV}$, respectively, corresponding to the oxidation state of gold metal. ${ }^{[31]}$ The UV/Vis spectra of $\mathrm{Au} / \mathrm{CeO}_{2}$ shows the absorption band centres at about $539 \mathrm{~nm}$ in wavelength owing to the LSPR effect; on the contrary, the $\mathrm{CeO}_{2}$ support itself shows no absorption in the visible light region, which substantially demonstrates that the contribution of light irradiation to affecting the catalytic activity and selectivity via the LSPR effect of Au NPs only.
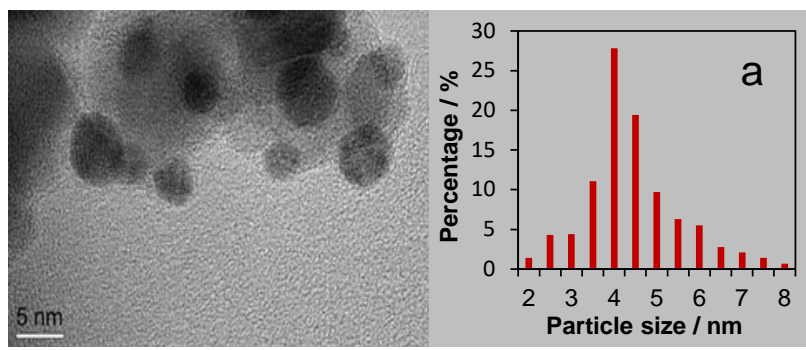

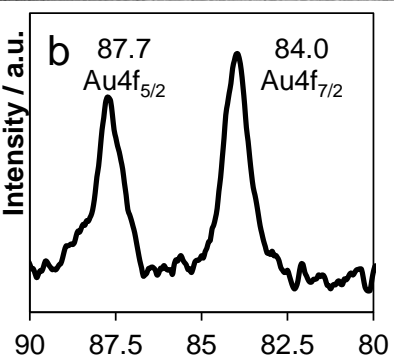

Binding energy $/ \mathrm{eV}$

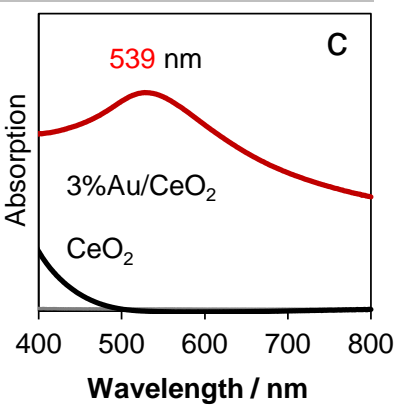

Figure 1. (a) TEM images of $3 \% \mathrm{Au} / \mathrm{CeO}_{2}$ with $\mathrm{Au}$ size distribution, (b) XPS spectra and (c) UV/Vis spectra of $3 \% \mathrm{Au} / \mathrm{CeO}_{2}$

It would be exciting if the underlying mechanistic reasons for the light irradiation which affects catalytic selectivity are unravelled. At the current stage of this study, we propose that the distinct differences of reaction pathway at a certain reaction temperature can be ascribed to "the reduction barriers of intermediate products" and "the light-induced electrons interaction" between reactant molecules and Au NPs, and these proposed associations have been initially explored in our previous studies. [24], [25], [32]

As the light irradiation essentially affects the catalytic reaction procedure, then it is sensible to deduce that the catalytic performances should differ with light source, light absorption and energy transfer. It is shown in Figure $2 \mathbf{a}$ that the selectivity towards azoxybenzene, azobenzene and aniline varies with the function of light wavelength under the same light intensity. But the ability of incident light to excite energetic electrons decreases with longer wavelength and thus the reaction activity declined. The excited electron distributions are different even with the same (macroscopic) light intensity. In general, with the shorter wavelength, there have more electrons in high energy level. For instance, with the same light absorption, at $505 \mathrm{~nm}$ and $582 \mathrm{~nm}$, the conversions are roughly the same, but the reaction proceeds to different stages, namely, the selectivity is different. In the case of $460 \mathrm{~nm}$ and $623 \mathrm{~nm}$, the latter only proceeds at the initial stage, having a $>99 \%$ of selectivity towards azoxybenzene (P1).

Besides light wavelength, the light intensity also has influence on the catalytic selectivity as shown in Figure $\mathbf{2 b}$. Increasing light intensities at the fixed wavelength (at $539 \mathrm{~nm}$ ) boost more energetic electrons, thus resulting in different product selectivity; the conversions also increase and undergo latter stages, that is, much higher yields of azobenzene (P2) and aniline (P3) are obtained.
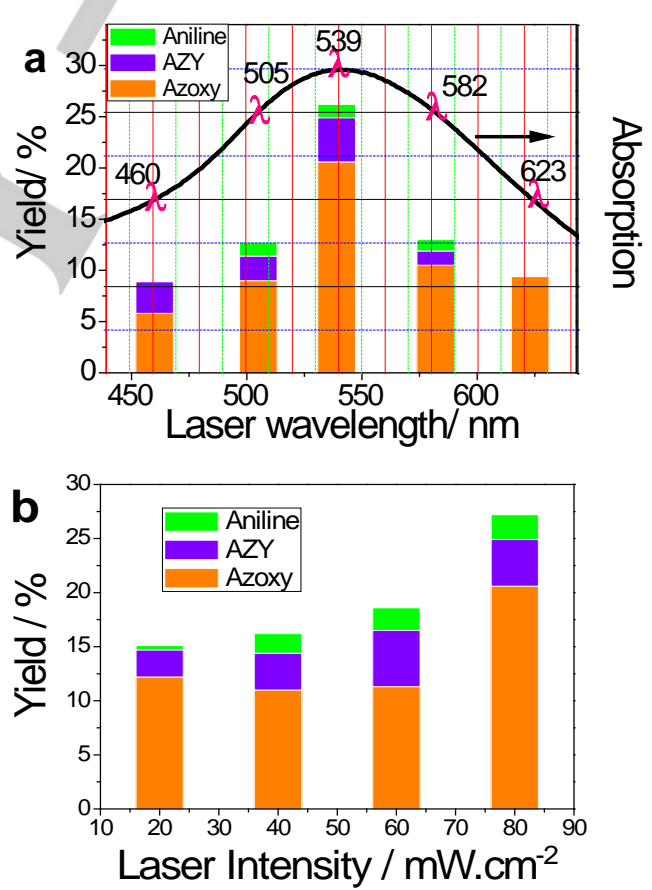

Figure 2. (a) The change in selectivity with the laser wavelength under the same light intensity of $80 \mathrm{~mW} \mathrm{~cm}^{-2}$; (b) the change in the product selectivity with the laser intensity at the same wavelength of $539 \mathrm{~nm}$.Notes: AZY refers to azobenzene, Azoxy to azoxybenzene.

Table 2.Catalytic performances of $3 \% \mathrm{Au} / \mathrm{CeO}_{2}$ for the selective oxidation of benzyl alcohol under simulate light source.

\begin{tabular}{|c|c|c|c|c|c|c|}
\hline \multirow{2}{*}{$\mathrm{T} /{ }^{\circ} \mathrm{C}$} & \multirow{2}{*}{ Light } & \multicolumn{2}{|c|}{ Selectivity (\%) } & \multirow{2}{*}{ Conv. ${ }^{c} / \%$} & \multicolumn{2}{|c|}{ Net change / $\%$} \\
\hline & & $\mathrm{Q} 1^{\mathrm{a}}$ & $\mathrm{Q} 2^{\mathrm{b}}$ & & Conv. & Q2 \\
\hline 40 & on & $>99$ & -- & 90 & 31 & -- \\
\hline
\end{tabular}

This is a post-peer-review, pre-copyedit version of an article published in Catalysis letters. The final authenticated version is available online at: https://doi.org/10.1007/s10562-018-2320-9 


\begin{tabular}{rllllll} 
& off & $>99$ & -- & 59 & & \\
60 & on & 91 & $\mathbf{9}$ & 97 & 28 & 9 \\
& off & $>99$ & -- & 69 & & \\
80 & on & -- & $>99$ & $>99$ & 6 & 93 \\
& off & 93 & 7 & 93 & & \\
\hline
\end{tabular}

a 1 1=benzaldehyde, ${ }^{\mathrm{b}} \mathrm{Q} 2=$ Benzoic acid, ${ }^{\mathrm{c}}$ Conversion. Reaction conditions: Reaction time $(6 \mathrm{~h})$. Solvent of isopropanol $30 \mathrm{ml}$, catalyst $(100 \mathrm{mg})$, benzyl alcohol $(3 \mathrm{mmol}), \mathrm{KOH}(0.1 \mathrm{M}, 3 \mathrm{ml})$, light wavelength (Full), and light intensity $\left(100 \mathrm{~mW} \cdot \mathrm{cm}^{-2}\right)$. Blank experiments: no products were detectable without catalysts or using the $\mathrm{CeO}_{2}$ support as catalysts under light on and off.

Further to test the generality of this finding that light irradiation can tune the product selectivity, the selective oxidation of benzyl alcohol to benzaldehyde and then to benzoic acid was also investigated, and the catalytic results are shown in Table 2. It is found that the light irradiation plays a role of switch in tunning the selectivity of benzaldehyde and benzoic acid at $80^{\circ} \mathrm{C}$. This result is inspiring because it not only proves the "switch role" of light irradiation for these two selected representative cascade reactions but also demonstrates that this phenomenon applies to both reductive and oxidative cascade reactions. At current stage, we do not have concrete evidence to uncover the tuning mechanism by light illumination, and it will be intriguing if some determining factors or correlations can be identified, for instance, illumination changes the donating or accepting behaviours of photoexcited electrons pathways, the reduction or oxidation pathways of reactions, or changes the binding energy or the redox potential of intermediate products on electronically polarized Au nanoparticles, and the like.

To conclude, this study highlights the contribution of light irradiation to tuning the selectivity of redox cascade reactions by means of supported plasmonic gold photocatalysts. The reduction of nitrobenzene and the oxidation of benzyl alcohol exhibit different selectivity with light on and off at a certain temperature. The net contribution correlates to light wavelength and intensity. To further identify or even quantify the contribution of light irradiation and to clarify their underlying mechanisms, more investigation will be performed on such as photocatalysts with different gold loadings or sizes and other redox cascade reactions. Overall, this study presents a promising approach to refining the product selectivity in clean chemicals synthesis, and could inspire the design of new catalytic materials and processes.

\section{Experimental Section}

Raw materials: Chemicals of chloroauric acid (99.999\%, $\left.\mathrm{HAuCl}_{4}\right)$, LLysine $(98.0 \%)$, sodium borohydride $\left(99.99 \%, \mathrm{NaBH}_{4}\right)$, hydrochloric acid $(37 \%, \mathrm{HCl})$, nitrobenzene $(99.0 \%)$, benzyl alcohol $(99.0 \%)$, potassium hydroxide $(99.0 \%, \mathrm{KOH})$, isopropanol (99.5\%), and azobenzene (98.0\%), were purchased from Sigma-Aldrich. The inert gas of argon was from the BOC (99.99\%). The supports of cerium
(IV) oxide (nanopowder, 99.95\%, $\mathrm{CeO}_{2}$ ) was also purchased from Sigma-Aldrich and without further purification.

Catalyst preparation: In a glass beaker with a magnetic stirring bar, $2.5 \mathrm{~g}$ of support powders $\left(\mathrm{CeO}_{2}\right)$, were dispersed into the 100 $\mathrm{ml}$ of $3.8 \times 10^{-3} \mathrm{M} \mathrm{HAuCl}_{4}$ aqueous solution. Subsequently, $20 \mathrm{ml}$ of $0.53 \mathrm{M}$ lysine was added dropwise to the mixture under stirring, and after that the stirring was prolonged for $30 \mathrm{~min}$. To this suspension, $10 \mathrm{ml}$ of $0.35 \mathrm{M} \mathrm{NaBH}_{4}$ newly-prepared solution was added dropwise, followed by adding $10 \mathrm{ml}$ of $0.3 \mathrm{M}$ hydrochloric acid (to achieve a $\mathrm{pH}=9.5$ ). The mixture was stirred for $1 \mathrm{~h}$ and aged for $24 \mathrm{~h}$. Then the solid was separated, washed firstly with 600 $\mathrm{ml}$ deionized water for three times in a $250-\mathrm{ml}$ centrifuge bottle to remove the chlorine ions, and the residual solution was tested by $0.1 \mathrm{M} \mathrm{AgNO}{ }_{3}$ solution and no $\mathrm{AgCl}$ precipitate was observed. The solids were collected, dried at $60^{\circ} \mathrm{Cfor} 16 \mathrm{~h}$, and used directly as the photocatalyst.

Photocatalytic reduction test: The reaction was conducted in a $50 \mathrm{~mL}$ round-bottomed Pyrex glass flask with a sealed spigot and a magnetic stirrer. The reaction temperature was controlled by an air-conditioner within a sealed wooden box. The light intensity in the reaction position was set at $0.1 \mathrm{~W} \cdot \mathrm{cm}^{-2}$ and could be adjusted by changing the distance between the reactor and the light source. The wavelength range was tuned by using various glass filters to cut off the irradiation below a certain value of wavelength. A tunable laser source (OPOTECK VIBRANT, $350 \mathrm{II}$ ) and a simulate solar source (NEWPORT, $150 \mathrm{w}$ ) were used as light source, respectively.

Catalytic reduction of nitrobenzene was conducted under the argon atmosphere. The experimental procedure was according to our previous study with slight modification. ${ }^{[33]}$ Typically, $3 \mathrm{mmol}$ nitrobenzene, $30 \mathrm{ml}$ isopropanol as solvent, and $3 \mathrm{ml}$ of $0.1 \mathrm{M} \mathrm{KOH}$ solution in isopropanal were mixed in the reactor, followed by adding $100 \mathrm{mg}$ of the catalyst and purging with argon gas, and then stirred during reaction and illuminated with the light source.

Catalytic oxidation of benzyl alcohol was conducted under the oxygen atmosphere. This experimental procedure was also based on our previous study with slight modification. ${ }^{[24]}$ Typically, $3 \mathrm{mmol}$ benzyl alcohol was dissolved into $30 \mathrm{ml}$ isopropanol, and $3 \mathrm{ml}$ of 0.1 $\mathrm{M} \mathrm{KOH}$ solution in isopropanal and $100 \mathrm{mg}$ of the catalyst were added into the mixture. The mixture was stirred during reaction and illuminated with the light source.

During these reactions, $0.5 \mathrm{ml}$ aliquots were collected at given irradiation time intervals and filtered through a Millipore filter (pore size $0.45 \mu \mathrm{m}$ ) to remove the catalyst particulates. Then flask was purged with argon again for more than $3 \mathrm{~min}$ to remove air and then sealed. The filtrates were analyzed by an Agilent 6890 gas chromatograph with the HP-5 column. Quantities of the products and reactants were calculateded from the peak areas of the compounds using calibration curves. An Agilent HP5973 mass spectrometer was used to determine and analyze the product compositions.

Characterization: TEM images were taken with a JEOL JEM-2100 transmission electron microscopeemploying an accelerating voltage of $200 \mathrm{kV}$. The specimens were sample powders deposited onto a copper microgrid coated with a holey carbon film. The element composition of some samples was determined by energy-

This is a post-peer-review, pre-copyedit version of an article published in Catalysis letters. The final authenticated version is available online at: https://doi.org/10.1007/s10562-018-2320-9 
dispersive X-ray spectroscopy attached on an FEl Quanta 200 scanning electron microscopy (The average gold loading was $2.8 \% \mathrm{wt})$. The diffuse reflectance UV-Visible spectra of the samples were recorded on a Cary 5000 spectrometer. The XPS data were recorded on an ESCALAB 250 spectrometer and Al Ka radiation was used as the X-ray source. The C1s peak at $284.5 \mathrm{eV}$ was used as a reference for the calibration of the binding energy scale.

Keywords: plasmonic effect $\bullet$ gold $\bullet$ cascade reactions $\bullet$ selective redox

[1] B. Smit, T. L. M. Maesen, Nature 2008, 451, 671-678.

[2] M. J. Climent, A. Corma, S. Iborra, Chem. Rev. 2011, 111, 1072-1133.

[3] J. Sun, A. M. Karim, X. S. Li, J. Rainbolt, L. Kovarik, Y. Shin, Y. Wang, Chem. Commun. 2015, 51, 16617-16620.

[4] A. M. Faisca Phillips, A. J. L. Pombeiro, M. N. Kopylovich, ChemCatChem 2017, 9, 217-246.

[5] J. Fan, M. De bruyn, V. L. Budarin, M. J. Gronnow, P. S. Shuttleworth, S. Breeden, D. J. Macquarrie, J. H. Clark, J. Am. Chem. Soc. 2013, 135, 11728-11731.

[6] R. A. F. Tomás, J. C. M. Bordado, J. F. P. Gomes, Chem. Rev. 2013, 113, 7421-7469.

[7] X. Zhang, K. Wilson, A. F. Lee, Chem. Rev. 2016, 116, 1232812368.

[8] C. M. A. Parlett, M. A. Isaacs, S. K. Beaumont, L. M. Bingham, N. S. Hondow, K. Wilson, A. F. Lee, Nat. Mater. 2016, 15, 178-182.

[9] J. Kou, C. Lu, J. Wang, Y. Chen, Z. Xu, R. S. Varma, Chem. Rev. 2017, 117, 1445-1514.

[10] H. Liu, T. Jiang, B. Han, S. Liang, Y. Zhou, Science 2009, 326, 1250-1252.

[11] C. O. Kappe, B. Pieber, D. Dallinger, Angew. Chem. Int. Ed. 2013, 52, 1088-1094.

[12] R. B. N. Baig, R. S. Varma, Chem. Soc. Rev. 2012, 41, 15591584.

[13] Y. Qu, X. Duan, Chem. Soc. Rev. 2013, 42, 2568-2580.

[14] H. Zhu, X. Ke, X. Yang, S. Sarina, H. Liu, Angew. Chem. Int. Ed. 2010, 122, 9851-9855.

[15] A. Marimuthu, J. Zhang, S. Linic, Science 2013, 339, 15901593.

[16] A. Tanaka, Y. Nishino, S. Sakaguchi, T. Yoshikawa, K. Imamura, K. Hashimoto, H. Kominami, Chem. Commun. 2013, 49, 2551-2553.

[17] X.-J. Yang, B. Chen, L.-Q. Zheng, L.-Z. Wu, C.-H. Tung, Green Chem. 2014, 16, 1082-1086.

[18] F. Wang, C. Li, H. Chen, R. Jiang, L.-D. Sun, Q. Li, J. Wang, J. C. Yu, C.-H. Yan, J. Am. Chem. Soc. 2013, 135, 5588-5601.

[19] L. Liu, S. Ouyang, J. Ye, Angew. Chem. Int. Ed. 2013, 52 , 6689-6693.

[20] X. Ke, S. Sarina, J. Zhao, X. Zhang, J. Chang, H. Zhu, Chem. Commun. 2012, 48, 3509-3511.

[21] S. Linic, P. Christopher, D. B. Ingram, Nat. Mater. 2011, 10, 911-921.

[22] P. Christopher, H. Xin, A. Marimuthu, S. Linic, Nat. Mater. 2012, 11, 1044-1050.

[23] K. Ueno, H. Misawa, J. Photoch. Photobio. C 2013, 15, 31 52.

[24] X. Zhang, X. Ke, H. Zhu, Chem. Eur. J. 2012, 18, 8048-8056.
[25] X. Zhang, A. Du, H. Zhu, J. Jia, J. Wang, X. Ke, Chem. Commun. 2014, 50, 13893-13895.

[26] M. Sankar, E. Nowicka, E. Carter, D. M. Murphy, D. W. Knight, D. Bethell, G. J. Hutchings, Nat. Commun. 2014, 5, 3332.

[27] A. Grirrane, A. Corma, H. García, Science 2008, 322, 16611664.

[28] Y. Takenaka, T. Kiyosu, J.-C. Choi, T. Sakakura, H. Yasuda, Green Chem. 2009, 11, 1385-1390.

[29] A. Corma, P. Concepción, P. Serna, Angew. Chem. Int. Ed. 2007, 46, 7266-7269.

[30] A. Grirrane, A. Corma, H. Garcia, Nat. Protocols 2010, 11, 429-438.

[31] O. P. Khatri, K. Murase, H. Sugimura, Langmuir 2008, 24, 3787-3793.

[32] X. Zhang, X. Ke, A. Du, H. Zhu, Sci. Rep. 2014, 4, 3805.

[33] X. Ke, X. Zhang, J. Zhao, S. Sarina, J. Barry, H. Zhu, Green Chem. 2013, 15, 236-244.

This is a post-peer-review, pre-copyedit version of an article published in Catalysis letters. The final authenticated version is available online at: https://doi.org/10.1007/s10562-018-2320-9 